\title{
Reliability Evaluation for LTE Based CBTC Train Ground Communication Systems
}

\author{
Shuo Wang $\mathbb{D}^{D}$, Li Zhu $(\mathbb{D}$, Kun Xu, Lin Zhang, and Xuan Wang \\ State Key Laboratory of Rail Traffic Control and Safety (Beijing Jiaotong University) 100044, Beijing, China \\ Correspondence should be addressed to Li Zhu; lizhu@bjtu.edu.cn
}

Received 27 February 2019; Revised 10 April 2019; Accepted 9 July 2019; Published 7 October 2019

Academic Editor: Zhi-Chun Li

Copyright (c) 2019 Shuo Wang et al. This is an open access article distributed under the Creative Commons Attribution License, which permits unrestricted use, distribution, and reproduction in any medium, provided the original work is properly cited.

\begin{abstract}
Nowadays, most communication based train control systems (CBTC) use Wireless Local Networks (WLAN) technology for information transmission. However, because of the high running speed of the train in urban rail transit, WLAN technology cannot fully satisfy the information transmission under the condition of train operation at a high speed. Long term evolution (LTE) technology has a high transmission speed, which can better meet the real-time requirements of train and ground information transmission. Therefore, LTE technology has become an emphasis on the research of communication technology in today's urban rail transit. Although there is a lot of research on the application of LTE technology in urban rail transit, there is little research on the reliability of LTE based train ground communication systems using experimental data. In this paper, the reliability of train ground communication is firstly defined. Then the train ground communication environment was established in the laboratory, and the performance parameters of train ground communication based on LTE technology were obtained in the test. Finally, the reliability is calculated and analyzed according to the experimental results, and a storage method of train data in actual operation is proposed, which can be used to analyze the reliability of train information transmission. The results show that the reliability of train ground communication based on LTE technology meets CBTC requirements.
\end{abstract}

\section{Introduction}

In the process of national economic development, rail transit played a significant role. Safe and efficient rail transit has laid a solid foundation for high-traffic passenger transport and high-speed freight transport. However, with the acceleration of China's urbanization process, people have put forward higher requirements for urban rail transit, and its reliability is the most important.

Urban rail transit possesses the characteristics of high speed and high tracing density. Nowadays, urban rail transit adopts CBTC systems as train control systems. The CBTC system can trace the train operation closely according to the situation of the forerunning train and the forward route. Hence, the minimum train tracking interval can reach the $90 \mathrm{~s}$ or less $[1,2]$, which can effectively shorten the train tracking interval and significantly improve transport efficiency [3].

The wireless communication system of the CBTC system must guarantee the reliable, safe, and prompt transmission of information. The high reliability of train-ground communication is a necessary precondition for the design of CBTC systems.

At present, urban rail transit systems adopt the wireless communication system based on WLAN. Engineering practice has proved that the current WLAN-based technology is one of the best technologies to achieve high security, high speed and high density of rail transit, but this technology also has some limitations. Considering the speed of rail transit, WLAN is not a communication mode designed for high-speed mobility, so it cannot meet the demand of urban rail transit, that is, the moving speed of users is more than $120 \mathrm{~km} / \mathrm{h}$. Therefore, transmission delay and packet loss are inevitable, which may lead to additional traction, braking, emergency braking, and the decrease of passenger satisfaction [6]. From the perspective of security, wireless LAN operates in the open frequency band, and many civilian devices also work in this frequency band, which is likely to cause interference. Finally, the WLAN cannot be scheduled according to the priority when the multi-service is concurrent, and the actual bandwidth of the high-priority service cannot be guaranteed, which 


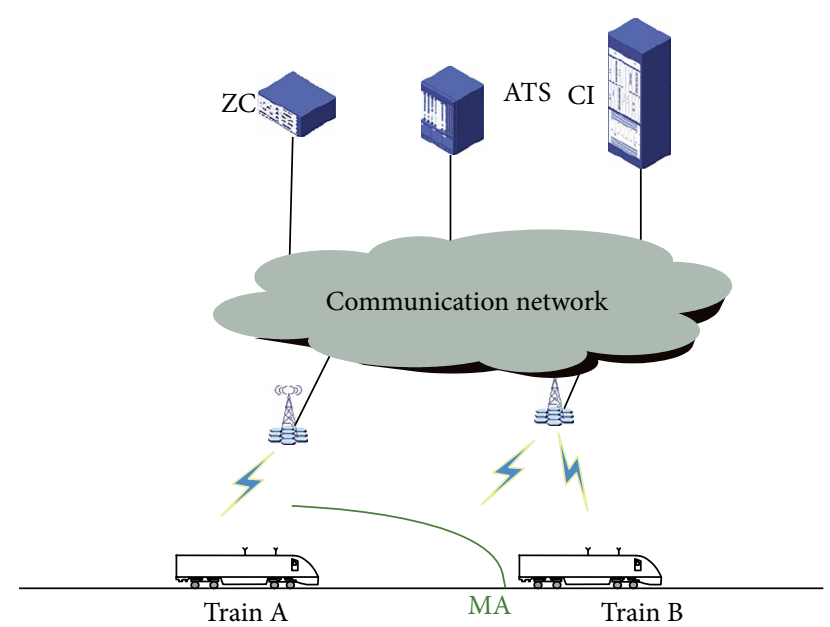

Figure 1: The structure of existing CBTC system.

is not conducive to ensuring the safe operation of the rail transit.

Nowadays the time-division-long-term evolution (TD-LTE) technology came into being in the urban rail transit system. For example, the newly opened Beijing Yanfang Line is based on TD-LTE. Possessing a series of advantages such as strong anti-interference capability and continuous coverage capability [7]. TD-LTE is a technical version of LTE that adopts time division duplexing.

LTE is a global common standard developed by the 3GPP (3rd Generation Partnership Project) based on OFDMA (Orthogonal Frequency Division Multiple Access) technology. As the mainstream of the fourth generation of communication technology, LTE can achieve faster data transfer rate and more stable handover. At the same time, LTE can reduce delay and packet loss rate effectively [8].

Compared with WLAN technology, LTE has a stronger advantage in urban rail transit [9]. There are main differences in spectrum resources between LTE and WLAN. LTE works in the licensed frequency band and needs to be employed on licensed spectrum resources. WLAN uses the same frequency band with bluetooth, ZigBee, and other systems without allocating frequency resources, and it is more susceptible to interference $[10,11]$. LTE supports flexible scheduling policies that can distinguish users from time and frequency and ensure the requirements of QoS [12]. However, the WLAN uses the mechanism of preemption and competition among users to schedule users. Resources are exclusively occupied by one user at a time. Therefore, collisions occur when the number of users is large enough, and the efficiency of resource utilization is reduced [13].

The introduction of LTE in the CBTC system can reasonably utilize the relatively low $1.8 \mathrm{GHz}$ frequency and the radio frequency leakage cable as the transmission medium, which can effectively enhance the reliability of the train-ground communication in the CBTC system [14]. Literature [15] introduced two crucial performance indicators of the packet loss rate and delay in the train communication system. Because of packet loss, if the control information is not able to be sent to train, the efficiency of train operation will be affected severely.
Also, the packet delay must also be controlled in a certain range. Otherwise, the information received by the train is not real-time information which makes a negative impact on the accuracy of train operation. In [16], literature studied the train control problem in CBTC systems of the wireless network.

China will use LTE-based wireless communication technology in a larger range of urban rail transit. Therefore, research on LTE communication technology in urban rail transit is particularly important. Although there have been many studies on LTE technology so far, few researches have been done to study the reliability of LTE based CBTC train ground communication systems based on experimental data.

In this paper, the reliability evaluation of the CBTC system based on LTE technology is carried out. The main content of this paper is as follows:

(1) We describe the framework and analyze the requirements of CBTC systems.

(2) Using the transmission delay and packet loss performance parameters, we define the reliability of the CBTC system.

(3) A real test environment is set up. We test the transmission delay and packet loss of the communication system, and the system reliability is evaluated.

The rest of this paper is organized as follows. Section 2 introduces the principle of CBTC and describes the requirements of train control systems and reliability definition of train ground communication systems. The testing environment based on the laboratory, which includes the environment of train-ground communication, is presented in Section 3. Section 4 is about the results of testing discussion and prospect.

\section{Safety-Critical Urban Transit Service}

2.1. The Principle of CBTC. The basic structure of the existing CBTC system is shown in Figure 1. It mainly includes data communication system (DCS), automatic train monitoring (ATS) system, computer interlocking (CI), zone controller (ZC), and vehicle controller (VOBC) [17]. The leading technologies of CBTC include [18]: (1) Wireless communication technology. (2) Mobile blocking technology. (3) Train positioning technology. During the train operation, ATS issues train-related driving plans to ZC through the backbone network. Then, the ZC establishes a movement authority (MA) based on the position report of all the traveling trains and the condition of the trackside controller and then transmits the MA to each train. The VOBC on each train controls the operation of the train based on the received MA.

Data Communication System (DCS) is one of the important subsystems for completing train-to-ground communication. And wireless communication system is an major component of DCS. When the CBTC system communication is in an unreliable situation, it will cause the data transmission delay to increase and the continuous packet loss phenomenon. Especially in automatic driving (ATO) mode, due to the unreliable communication of the train control system, the train 


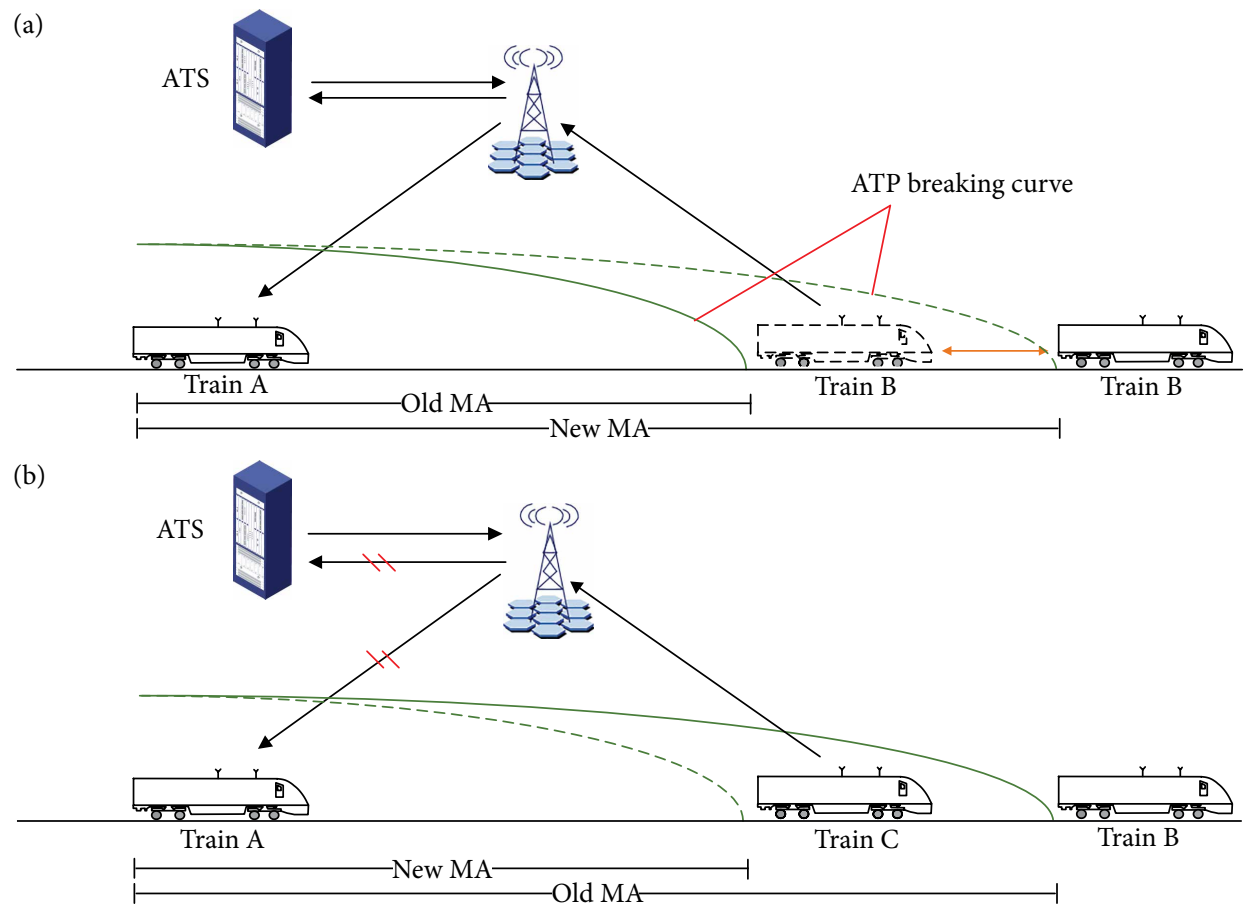

FIGURE 2: The impacts of communication interruption on CBTC systems.

may not receive the information of $\mathrm{MA}$ in time, causing unnecessary braking and traction [19]. Hence, reliability is an essential index in wireless communication systems. Train running on the track will always receive information from equipment on the ground and send messages to staffs. When the wireless communication link is not available when needed, trains may not be able to successfully obtain the control messages about operation plan from systems such as ATS or CI, and cannot send running state of train or alarm messages to dispatchers. It will have a significantly negative impact on train control performance. Therefore, the reliability of wireless communication systems needs to be defined and calculated to evaluate the CBTC system's performance.

2.2. The Requirements of CBTC. In CBTC systems, based on the position of all the trains and obstacles along the rail and the operation plan acquired from ATS, each train computes its MA. The real-time braking curve is calculated using the newest MA by ATP on the train. When the train's speed get to the limited velocity on the braking curve, the train will start decelerating to ensure that the speed of train will never exceed the warning line and travel out of its MA. The ATS sets the trip time between two stations. According to the trip time and some other performance indices, energy savings and passengers comfort, for example, and then the ATO derives an optimized guidance trajectory. The optimal velocity of the train in a specific position is obtained from the velocitydistance guidance trajectory. The main task of ATO is to make the train run according to this optimized train running curve.

Figure 2 shows the impacts of wireless communication reliability on CBTC systems performance. As shown in Figure 2(a), when the communication is available, the updated position of the Train B, whose position is located far from Train A in the figure, will be sent to the following trains in every communication period using the communication network. The following train will operate as the braking curve and will never exceed the MA point unless the MA is updated. However, when the communication interruption or delay in data communication systems is too substantial, the newest MA point may not be able to be sent to the following train by $\mathrm{ZC}$ for a certain period. As shown in Figure 2(b), before the updated MA arrives, the old MA is still used by the following train. When the ATP receives the updated position, it will calculate a new breaking curve and the train will come back to the new optimized guidance trajectory controlled by ATO. Compared to the scenario when communication is always available, the last arrived train position caused by communication interruption or delay makes the train travel deviate from the guidance trajectory. Substantial energy will be lost during this process, and it will severely affect passenger comfort. More importantly, it will increase the trip time between stations, which will affect the operation of all the trains behind.

2.3. Reliability Definition of CBTC. In the process of data transmission, transmission delay will be bound to happen, and because of channel fading and other adverse environmental factors, packet loss will also take place. In one case, just as shown in Figure 2(a), when Train B is in front of Train A, on account of transmission delay, when Train A gets the messages about operation plan and according to that to calculate MA, the actual MA is further than the MA that Train A has calculated considering that Train B is still running. And in the other case, as shown in Figure 2(b), when Train $C$ get into this line from another one, Train A has got a MA at the end of Train B and it needs to calculate a new MA at Train C. However, because of packet loss, Train A cannot receive the newest information. 


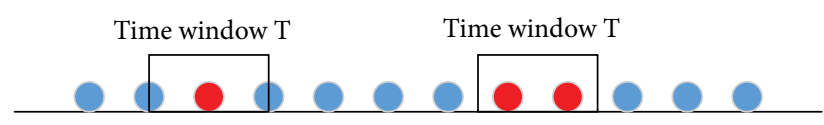

(a)

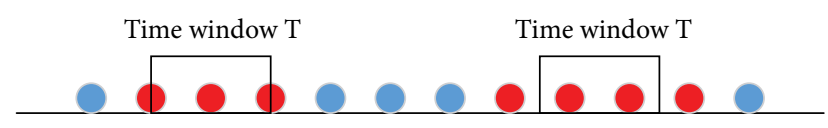

(b)

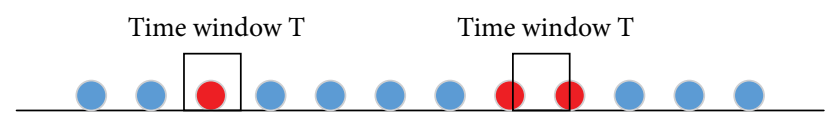

(c)

Packet received

Packet lost

FIGURE 3: Data communication system reliability definition.

If Train A still runs according to old MA, Train A will have a collision with Train C. It is against the requirements of CBTC.

According to the above description, we use transmission delay and packet loss to define the reliability of wireless communication system [20]. In the transmitting process, it is desirable to maintain the communication link between two communication terminals available within a specified period of $T$. Therefore, we define the data communication reliability in CBTC systems as the probability when one communication terminal can successfully receive information from the other communication terminal within the time window $T_{\text {window }}$. Reliability is divided into two levels, including delay level reliability and packet loss level reliability. At the same time, the reliability of the wireless communication system is calculated from these two levels of reliability.

Delay reliability is to measure the reliability of communication according to the transmission delay of packets, indicated by $P_{\text {delay }}$. It is defined as the percentage of packets that are delayed by no more than the time window and the total number of packets transmitted. When the transmission delay of a packet exceeds the specified time window $T_{\text {window }}$, it is considered that there is a problem in the information transmission of the packet. In CBTC systems, it is considered that data packets are exchanged at every communication period. When the time between two consecutive received data packets is greater than time window $T_{\text {window }}$, the data communication system is considered to be unreliable $\nmid$ the higher the number of packets whose delay exceed the time window $T_{\text {window }}$, the lower the reliability of communication system.

Packet loss reliability refers to the reliability of communications measured by the number of lost packets, represented by $P_{\text {loss }}$. It is defined as the percentage of the number of packets not affecting the communication and the total number of packets. In this paper, continuous packet loss duration can be obtained based on the data transmission rate and packet size. We believe that when the time taken by consecutive packets loss exceeds the defined time window $T_{\text {window }}$, packet loss will affect the reliable transmission of system information, and when the consecutive packets loss time is less than the time window $T_{\text {window }}$, the standard transmission of system information will not be affected.
In Figure 3, the dots of different colors in the figure indicate the data packets in the transmission, the blue dots indicate the packets successfully received by the receiving end, and the red dots indicate the data not successfully received by the receiving end. When the time window $T_{\text {window }}$ takes different values, the number of consecutive lost packets has different effects on the reliability of the system. Just like in Figure 3(a), the value of the time window is larger than the time for transmitting two data packets. Therefore, the loss of these two data packets does not affect the reliability of the system, and as defined, the loss of one data packet does not affect information transmission either. Moreover, in Figure 3(b), the value of the time window is less than the time for transmitting three data packets, so when three or more data packets are consecutive losses, the transmission of information is deemed to be affected. The more consecutive packets loss exceeds the time window $T_{\text {window }}$, the higher the $P_{\text {loss }}$, the lower the reliability of the train communication. As shown in Figure 3(c), the time window $T$ takes longer than the time it takes to transmit a packet. Loss of two packets will also affect the transmission of information.

The reliability of train communication is $P_{\text {system }}$, which consists of delay level reliability $P_{\text {delay }}$ and packet loss level reliability $P_{\text {loss }}$. The main parameters needed to measure the reliability of train communication $\left(P_{\text {system }}\right)$ are as follows:

(i) $T_{\text {window }}$ : The defined time window. When transmission delay or consecutive packets loss time exceeds the time window $T_{\text {window, }}$ it is considered that the transmitted data packet fails to reach the receiving end

(ii) $n u m_{\text {delay }}$ : The total number of packets whose delay is higher than the time window $T_{\text {window }}$

(iii) $n u m_{\text {loss: }}$ : The total number of dropped packets that the consecutive packets loss time exceeds the time window

(iv) $C_{\text {delay: }}$ The total number of packets sent during the delay level reliability test

(v) $C_{\text {loss: }}$ : The total number of packets sent during the packet-loss level reliability test

(vi) $P_{\text {delay }}$ : The ratio of the number of packets whose delay do not exceeds the time window $T_{\text {window }}$ to the total number of transmitted packets

(vii) $P_{\text {loss }}$ : The ratio of the number of consecutive dropped packets not over the time window $T_{\text {window }}$ to the total number of sent packets

(viii) $P_{\text {system }}$ : The reliability of train communications, consisting of delay level reliability and packet loss level reliability.

By definition, the delay level reliability is $P_{\text {delay }}=n u m_{\text {delay }} / C_{\text {delay }}$ and the packet loss level reliability is $P_{\text {loss }}=n u m_{\text {loss }} / C_{\text {loss }}$. So, the train communication reliability $P_{\text {system }}$ consisted of $P_{\text {loss }}$ and $P_{\text {delay }}$, is calculated from the results of the two tests based on the proportion of the total number of packets transmitted by the two tests. Therefore, the reliability formula for train communication $P_{\text {system }}$ can be derived as: 


$$
\begin{aligned}
P_{\text {system }} & =\frac{C_{\text {delay }}}{C_{\text {loss }}+C_{\text {delay }}} \cdot P_{\text {delay }}+\frac{C_{\text {loss }}}{C_{\text {loss }}+C_{\text {delay }}} \cdot P_{\text {loss }} \\
& =\frac{C_{\text {delay }}}{C_{\text {loss }}+C_{\text {delay }}} \cdot \frac{n u m_{\text {delay }}}{C_{\text {delay }}}+\frac{C_{\text {loss }}}{C_{\text {loss }}+C_{\text {delay }}} \cdot \frac{n u m_{\text {loss }}}{C_{\text {loss }}} .
\end{aligned}
$$

\section{Test Scenario and Configuration}

3.1. Test Environment. The CBTC system utilizes wireless communication to realize communication between the ground device and the onboard device and two-way large-capacity information transmission so that the ground device can timely transmit the speed limit condition of the front line and vehicle device can obtain the front line information in time. The running train calculates the optimal braking curve in real time and runs according to the running curve, thereby increasing the line capacity, reducing the frequent deceleration braking, improving passenger comfort and significantly improving the safety of train operation.

Nowadays, Researchers began to study the use of LTE technology as a communication technology for CBTC systems. LTE is a communication technology with a complete QoS transmission management strategy and higher stability than WLAN. Also, LTE communication technology has been used to transmit messages on some rail transit lines. Not only that, but scholars have begun to research and improve LTE technology for use in next-generation train control systems. Such as, in $[21,22]$, the author studied the use of CBTC system based on LTE-M with FlashLinQ T2T communication.

Just as shown in Figure 4, it adopts a flat network structure, including a three-layer architecture (EPC, E-UTRAN, and UE) and use IP transmission. The LTE-based wireless communication technology can realize timely and accurate transmission of information such as CBTC information, train status monitoring information, video surveillance (CCTV), and PIS (including emergency text), providing a solid foundation for the safe and efficient operation of urban rail transit systems.

Compared with GSM-R, LTE wireless communication technology has many advantages in rail transit. Some of the performance benefits are shown as follows:

(1) Latency. In the process of information transmission, the delay time of the UE to user plane of the eNodeB is less than $10 \mathrm{~ms}$, and the delay time of the control plane is less than $100 \mathrm{~ms}$ providing better conditions for real-time information transmission between ground control equipment and vehicle equipment.

(2) Throughput. At a Bandwidth of $20 \mathrm{MHz}$, the downlink peak data rate reaches $100 \mathrm{Mbps}$, and the upstream peak rate reaches $50 \mathrm{Mbps}$.

(3) Capacity. In the $5 \mathrm{MHz}$ bandwidth, LTE technology can support 200 active users, and when the bandwidth is in the range of $5 \mathrm{MHz}-20 \mathrm{MHz}$, it can support 400 active users, which fully meets the requirements for the maximum number of operating vehicles required on a railway line.

(4) Mobility. When the moving speed of the user is in the range of $15-120 \mathrm{~km} / \mathrm{h}$, it can have higher service

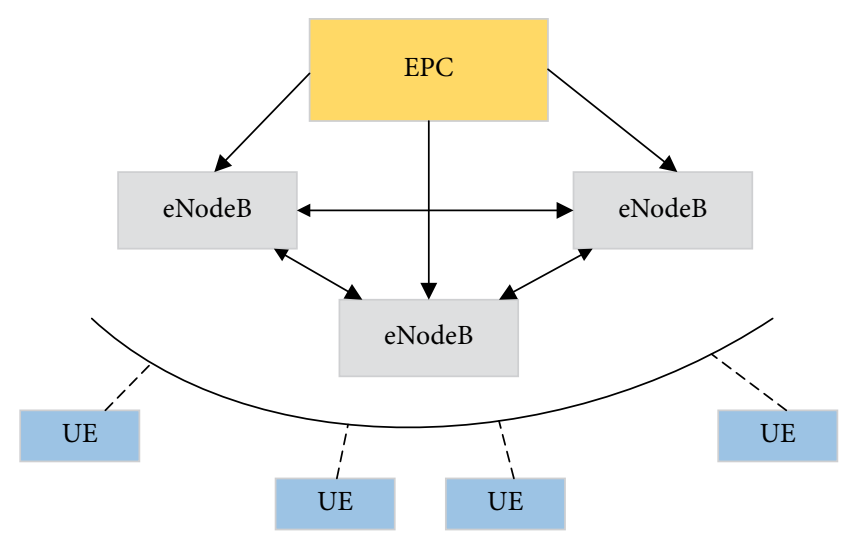

Figure 4: Architecture of LTE.

performance, and in the range of $20-500 \mathrm{~km} / \mathrm{h}$, it provides services of equal or better quality than 3GPPR6. Moreover, LTE technology supports the use of services not only under high-speed mobile conditions but also under low-speed conditions.

In commercial cell networks, 400 users can be activated under $5 \mathrm{MHz}$ bandwidth, but the quality of service for each user will be degraded significantly, which cannot be acceptable in the urban rail system. Indeed, in the real system, it is not recommended to activate more than 10 users when the bandwidth is $5 \mathrm{MHz}$. As is known, the bandwidth can be set as $1.4 \mathrm{MHz}$ in the LTE system. However, because of the integrated traffic, including train control information, PIS, CCTV, and other information, and limited throughput under this bandwidth configuration, the train control information is the only traffic that can be transmitted through the wireless communication system in CBTC.

Here, we built an indoor test environment at the State Key Laboratory of Rail Traffic Control and Safety at Beijing Jiaotong University. In the lab, we prepared the hardware devices needed for the test, including LTE devices for communication, as well as devices such as a programmable attenuator, fixed attenuators, and channel emulator. The connection of the device is as shown in Figure 5.

EB Propsim, as a channel simulator, simulates the attenuation, fading, time delay, and Doppler frequency shift characteristics of single-channel wireless transmission during train operation using precomputation files to simulate the transmission environment. A fixed attenuator is used to adjust the intensity of the signal received by TAU.

3.2. Test Procedure. Regarding the test, first, we set the parameters we need. In this lab, we chose to test the CBTC service as a test service and use IxChariot to simulate service performance testing and install it on the server and client to complete the basic performance test of LTE. According to the business requirements of the CBTC in the signal system, the data packet of the service flow adopts the UDP protocol. Moreover, the data packet size is set to 400 bytes. The data transmission rate is $256 \mathrm{kbps}$ downstream, and the uplink is $256 \mathrm{kbps}$ (the statistical interval of the test indicator is 1 second). 
In an LTE network, different bandwidths are set for transmitting CBTC services under different conditions. However, in this test, since the CBTC service transmits a relatively small amount of traffic, a transmission bandwidth of $1.4 \mathrm{MHz}$ is selected. Moreover, because the train-ground communication system can better accomplish transmission tasks with higher bandwidth, choosing a smaller bandwidth to analyze its reliability can indirectly indicate that the train-ground communication system is more reliable at greater bandwidth.

At the receiving end, the signal strength of the ground information that the train can receive is different during the actual running of the train, and the data transmission is better when the signal strength is large. Therefore, the test selected two static signal models for discussion, including near-point and far-point models. We specify that the RSRP of the signal is less than $75 \mathrm{dBm}$ at the near point and the RSRP range of the far point is $[-85,-90] \mathrm{dBm}$.

To simulate the channel environment during train operation and to test LTE-based train communication capabilities in high-speed operating environments, we used the EB Propsim channel simulator. The load channel model has a train speed of $200 \mathrm{~km} / \mathrm{h}$, using the ITU-VA channel model and standard Doppler spectrum.

Particularly, in the latency test, the packet size was set to 400 bytes, and 2000 packets were tested. We need to add a timestamp to the analog service data packet sent by the sender and calculate the delay by analyzing the timestamp of the analog service data packet received on the receiving endpoint. In this experiment, the data was processed before the analysis. The response time data obtained in the experiment is the round-trip response time of the communication between the train and the ground. We do not consider the environment, equipment and other problems in the actual scene. Here, our default transmission response time is equal to the received data response time.

The following are the test steps for communication between the train and the ground terminal. Before starting the communication test between the train and the ground terminal, first connect the device according to the connection method shown in the Figure 5, and confirm that the unit can be set normally, and the terminal can search the network. Then we need to load the channel model on the channel simulator and plug in the fixed attenuator. Finally, open the IxChariot script on the vehicle analog terminal, correctly load the CBTC service and set the transmission parameters as described above. When we are ready, we can start testing. First record the device model, software version, RSSP, SINR, system bandwidth, AMC adaptive parameters and whether ICIC is enabled. After that, start the service simulation software and load the one-way CBTC service. In the delay test, the test lasted for $5 \mathrm{~min}$, while in the packet loss test, the test lasted for $55 \mathrm{~min}$. Test and record the transmission delay and total packet loss rate of the uplink and downlink packets.

\section{Test Results and Discussions}

4.1. Test Results. In this section, we give the test results of the train-ground communication system based on LTE technology, tested in the laboratory, including the RTT transmission response time and the wireless network packet loss rate, and calculate the reliability of the train-ground communication system.

This article will analyze the transmission performance under the $1.4 \mathrm{MHz}$ bandwidth in detail, and calculate the reliability of the train-ground communication system under the experimental environment conditions. In this experiment, Figure 6 is the transmission response time chart of a CBTC service at the near point, and Figure 7 is the transmission response time chart of a CBTC service at far point. As can be seen in Figures 6 and 7, the data transmission time lasts 5 minutes, and the round-trip response time of train-ground communication is less than $300 \mathrm{~ms}$, which indicates that the train-ground communication system based on LTE technology can transmit information stably. It also can be seen from figures that the response time of the near point is less than the response time of the far point.

Figure 8 shows packet loss rate charts of CBTC business at $1.4 \mathrm{MHz}$ bandwidth. In this figure, we can see that the packet loss rate test lasts for 55 minutes. During the data transmission process, there is a certain amount of data loss. And during the entire data transmission process, the loss of data packets is affected by real-time changes of environment and performance of equipment.

Figure 9 shows the continuous packet loss rate of the CBTC service. It can be seen that there is a continuous loss of different numbers of packets. According to the different transmission information, there are different influences on the communication between the train and the ground. In this experiment, whether the lost data packet will affect trainground communication is determined by $T$ value. The higher the $T$ value is, the less the impact on train ground communication. In this experiment, after statistics, there are only two or three consecutive packet losses at the near point. While at far point, there exist more than four consecutive packet loss.

Figure 10 shows the reliability of delay-level at the near point and far point. Figure 11 shows the reliability of packet loss level at the near point and far point. According to the definition, the reliability of delay level and packet loss level are obtained under different time windows. Data packets with delays longer than $T$ are excluded, and they are considered to have failed to be transmitted successfully. If consecutive packet losses occur, the continuous packet loss time does not exceed the time window $T$ at this time, and it is considered that it does not affect the data transmission. As can be seen from the Figure 10, the reliability of the delay level and the reliability of the packet loss level both reach a certain value, and after the time window reaches a certain length, it can maintain a relatively stable value. Also, from the figure, we can see that when we take the same time window $T$, the reliability of the near point model is higher than that of the far point model in terms of transmission delay and packet loss rate.

Figure 12 shows the reliability of the train-ground communications system at different $T$ values and different static models. According to the data processing method described in the previous section, the reliability of the train-ground communication system is calculated based on the reliability of the transmission delay level and the reliability of the packet loss 


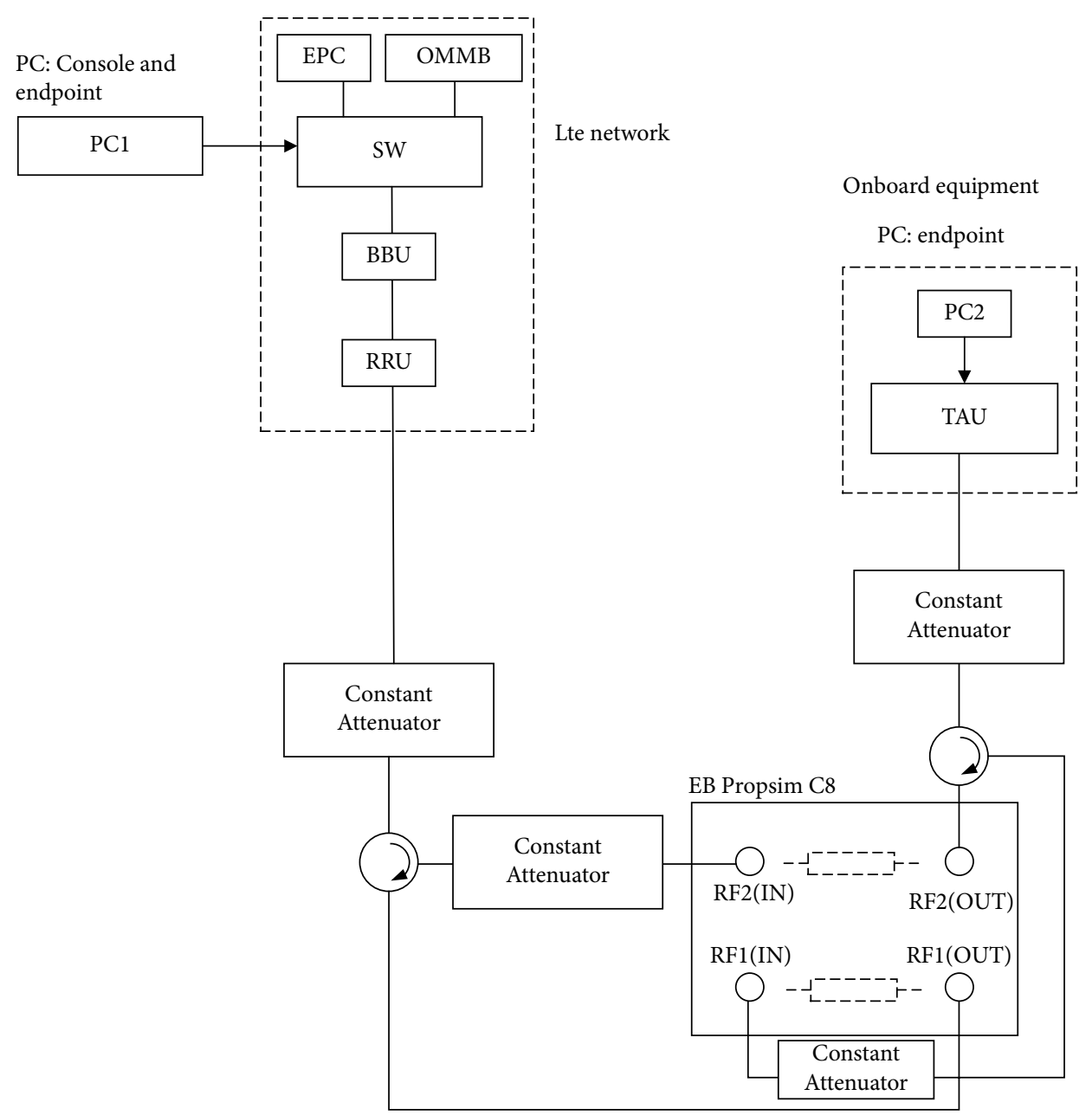

FIGURE 5: Communication test link between train and ground terminal.

level. It can be seen from the figure that the increase of the $T$ value can improve the reliability of the train-ground communication system. The reliability can reach over $80 \%$, and the reliability of the train-ground communication system is related to the reliability of the transmission delay level and packet loss level. The reliability of the train-ground communication at the near-point model is higher than that at the far-point model.

4.2. Discussion and Prospect. From the above test results, it can be seen that the transmission delay and packet loss of train-ground communication remain below a certain level regardless of the bandwidth. Also, we can see that even under an unfavorable configuration which are at $1.4 \mathrm{MHz}$ bandwidth and low RSRP, the train ground communication system still has high reliability, indicating that LTE technology can complete the communication of train and ground and meet the requirements of the urban rail transit system. At the same time, due to the limitations of the field test environment, there is strong signal interference. However, reliability is still good. The result shows that under unfavorable environmental conditions, LTE technology can also complete the data transmission between train and ground in a high velocity and accuracy ensuring the safe and efficient operation of urban rail transit. Therefore, we conclude that LTE technology applies to the train-ground communication system in urban rail transit. Also, it has extremely high reliability, indicating that it is sufficiently suited for transmitting the information of the event recorder.

During the operation of the train, due to the mutual electromagnetic interference between the equipment in the subway system and the influence of the natural environment, or the improper operation of human beings, the train may have faults and accidents. At this point, the analysis and evidence collection of the cause of the accident is particularly essential. Therefore, a train event recorder capable of recording information in real time is required to record the operating status of a various device, the real-time information of the train operation, and the related operations of the train staff during the running of the train.

In the 1990s, the Institute of Electrical and Electronics Engineers specifically described the train event recorder in relevant technical documents. Also, in the RAILROAD LOCOMOTIVE SAFETY STANDARDS in the United States, the event recorder is clearly defined: (CFR 49 Ch II 229.5): "Event recorder means a device, designed to resist tampering, that monitors and records data." The event recorder will completely record the train-triggered status and various operational information during the continuous change as shown below: 


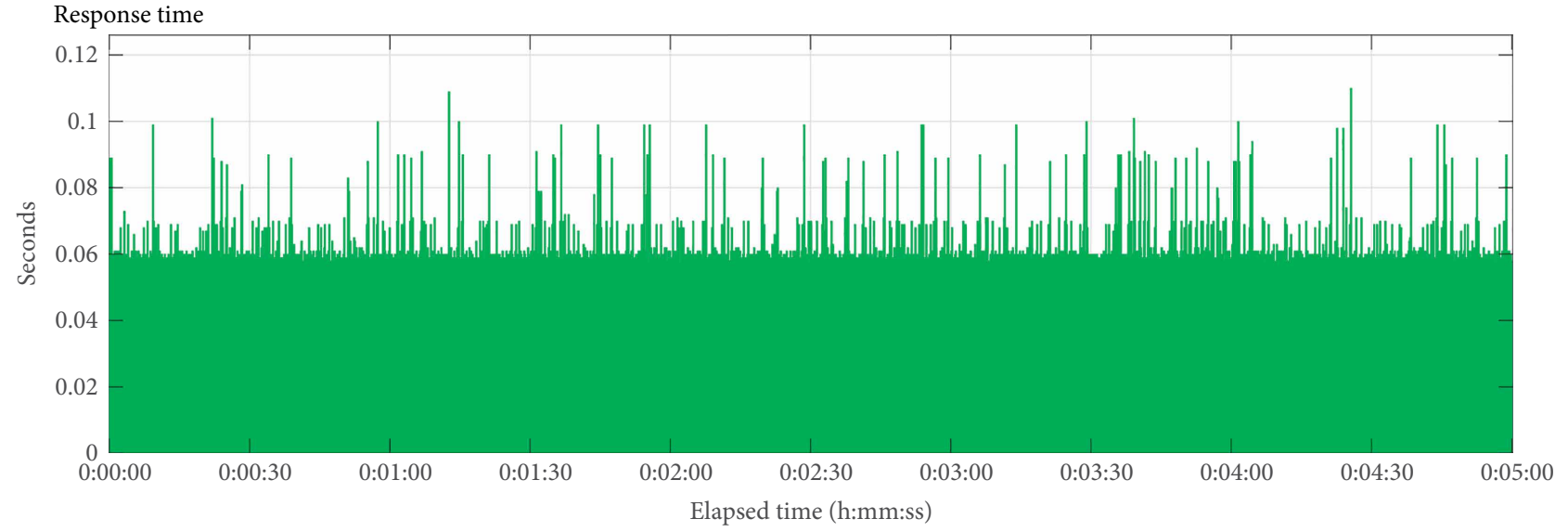

FIgURE 6: Response time of the near point.

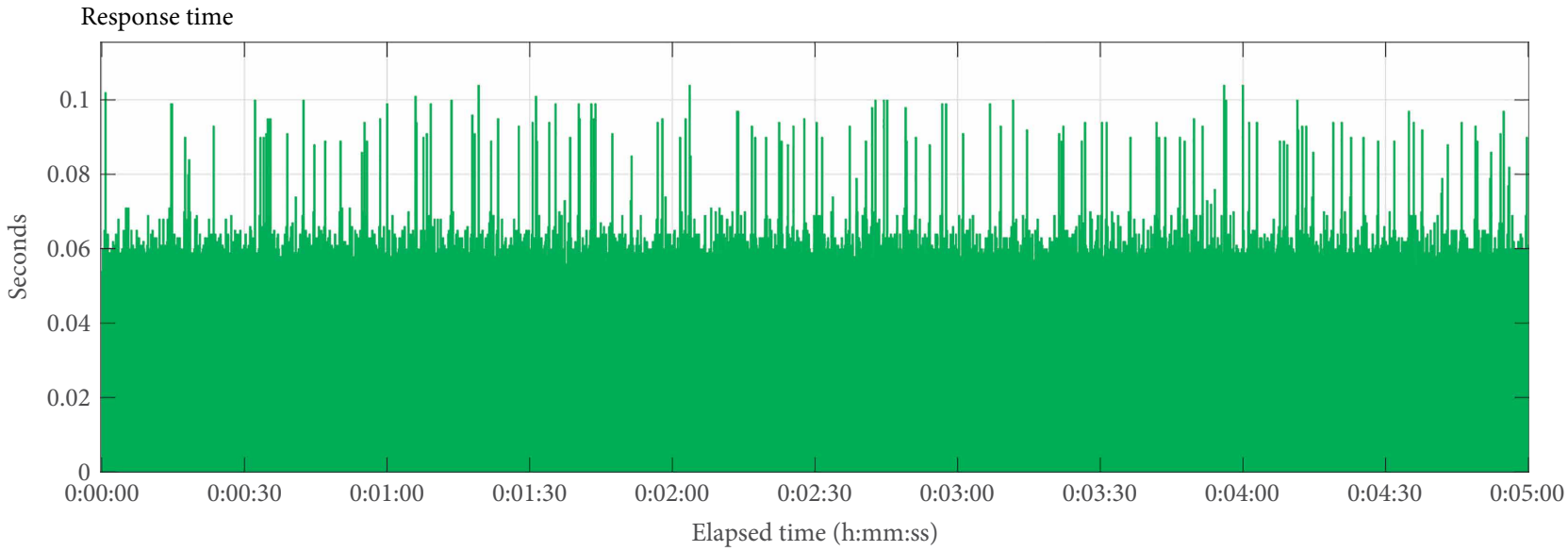

FIgURE 7: Response time of the far point.

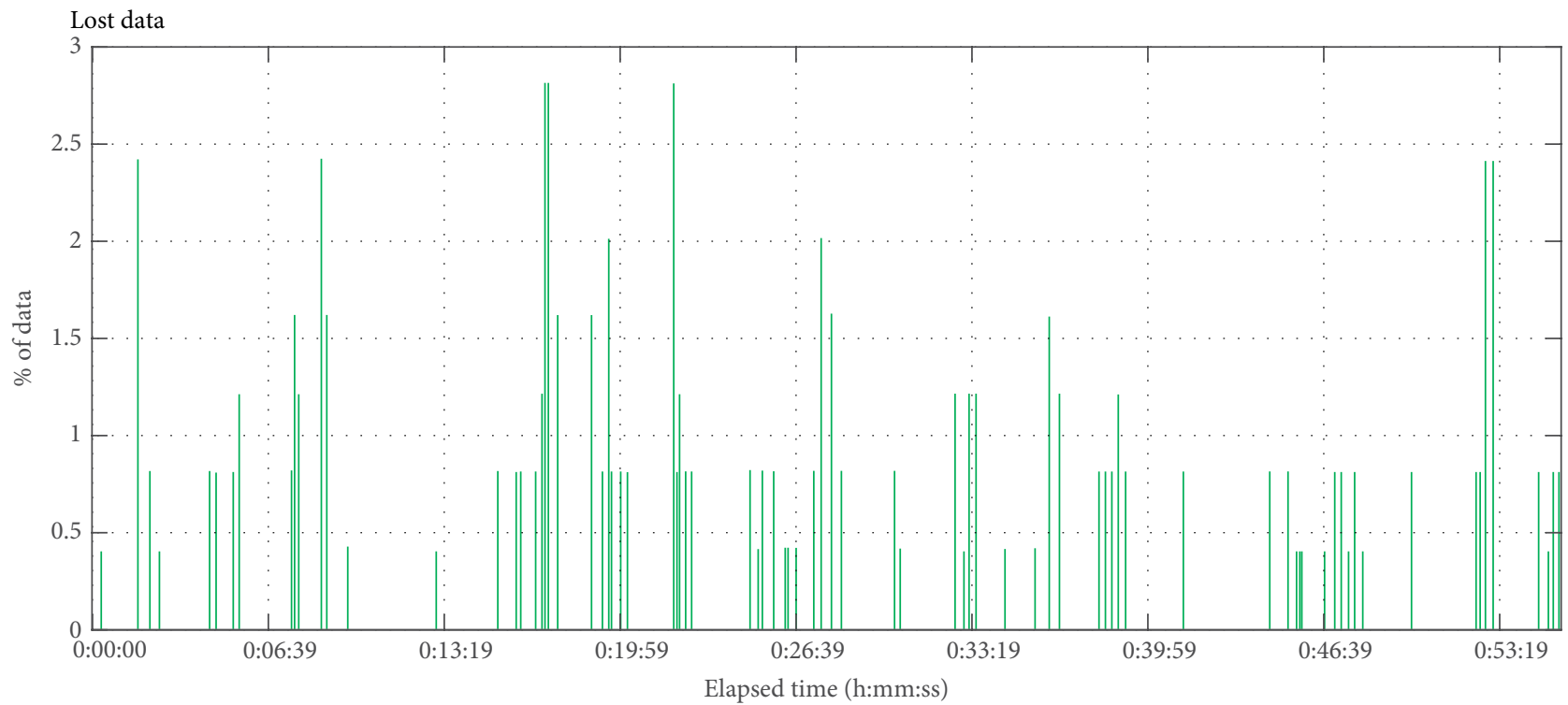

FIGURE 8: Lost data. 


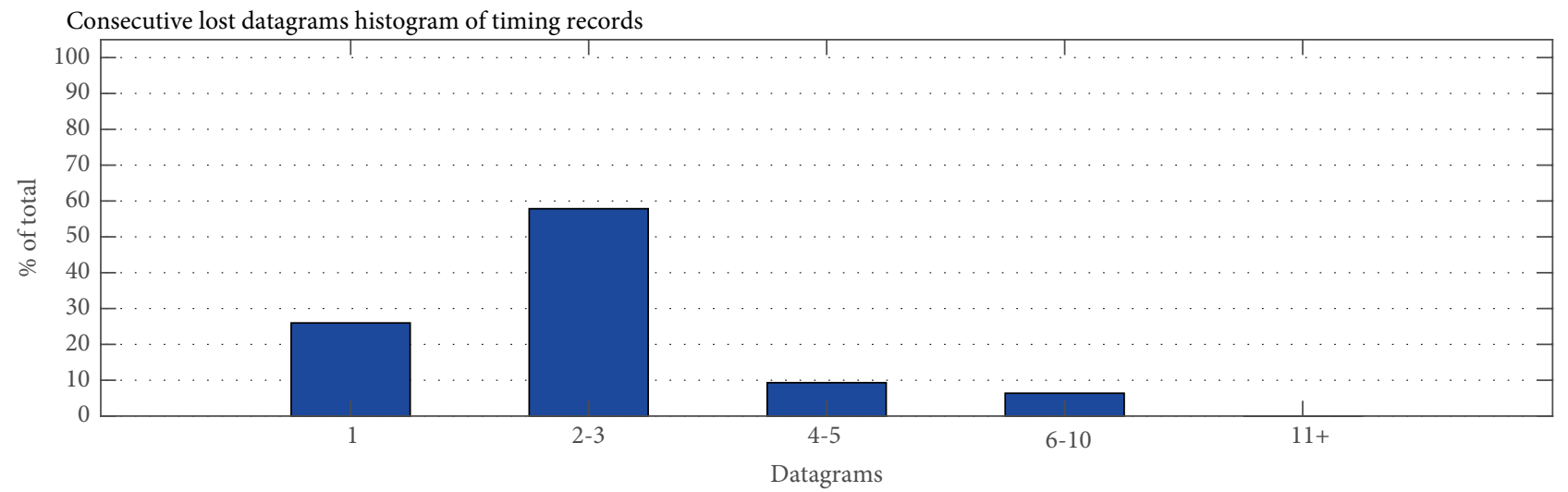

FIGURE 9: Consecutive lost datagrams histogram of timing records.

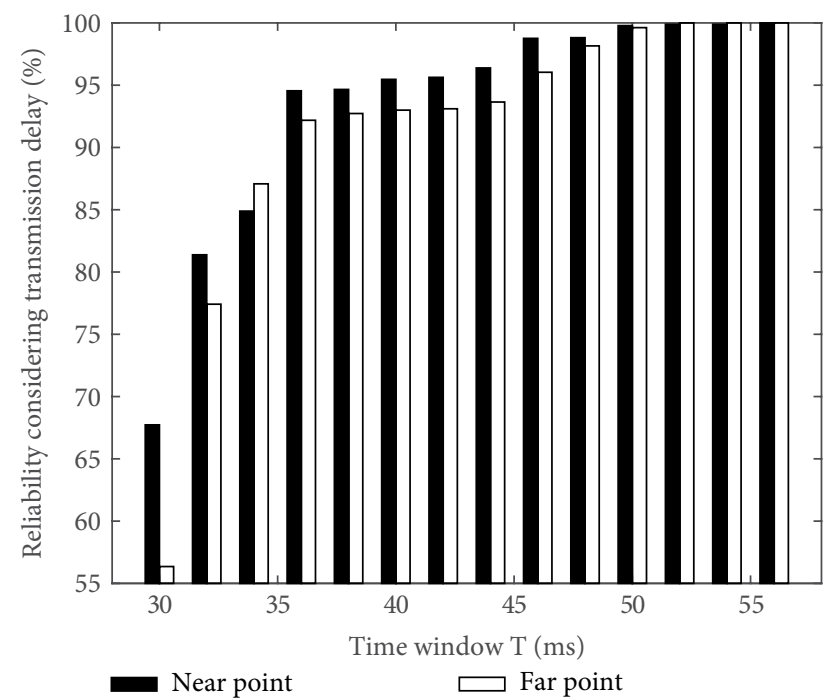

FIGURE 10: Reliability considering transmission delay.

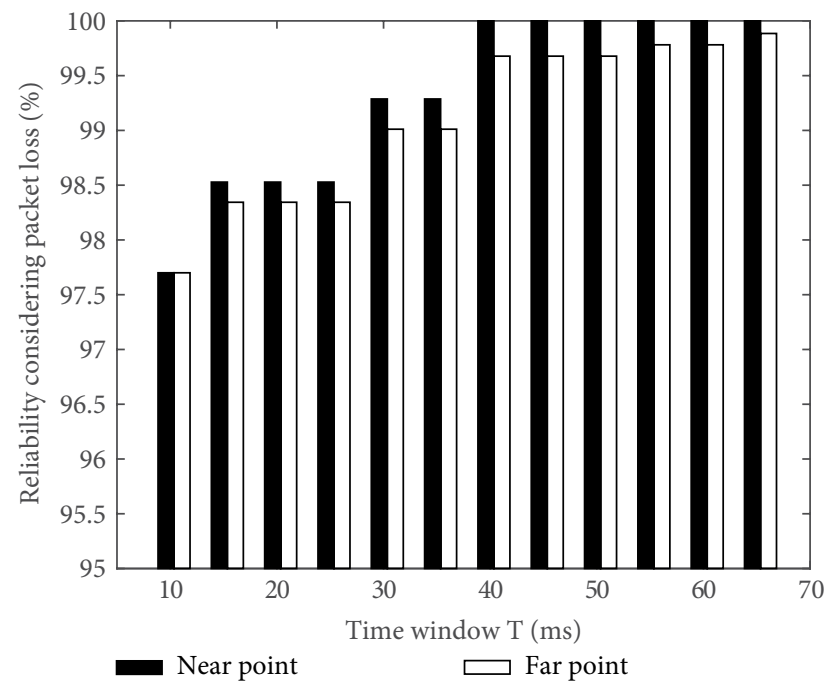

FIGURE 11: Reliability considering packet loss.

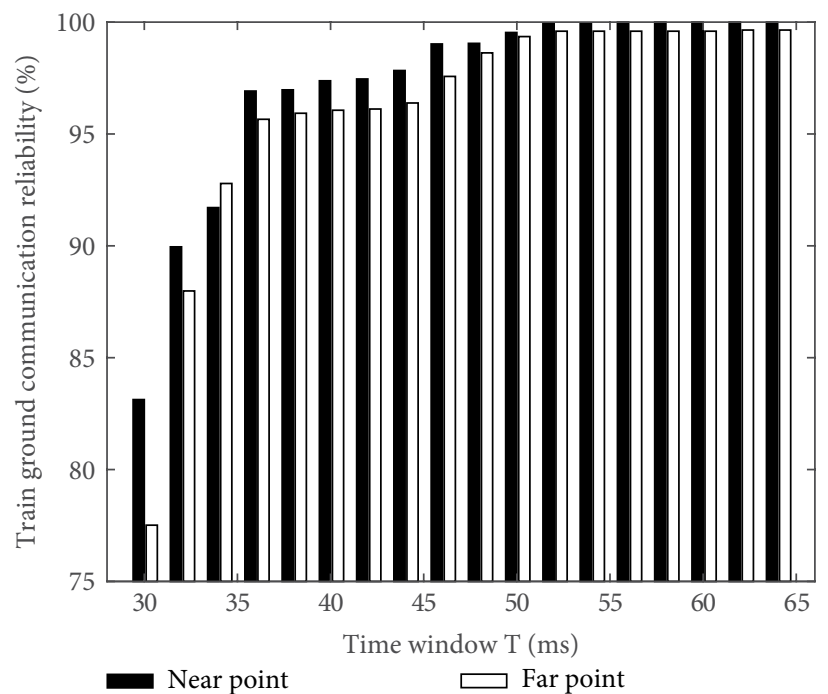

FIGURE 12: Reliability of train ground communication system.

(1) The real-time operation status of the train. Speed, running direction, running distance, etc.

(2) Train equipment operating status.

(3) Man-made control operations performed by operators. Brake, accelerate, decelerate, etc.

After the event recorder stores the information, whether the data can be obtained effectively is of great significance. In the event of a train accident, there is a high probability that the incident recorder will be severely damaged, so that the stored data will not be accessible to accident investigators. Therefore, how to save the acquired information well becomes a necessary premise for analyzing the cause of the accident.

According to the characteristics of information transmission in rail transit, we propose an storage scheme based on the existing information storage methods. Using LTE communication technology, all equipment information and operating status related to locomotive operation during train operation are sent to the cloud storage space through the wireless communication system in real time. Therefore, the ground staff 


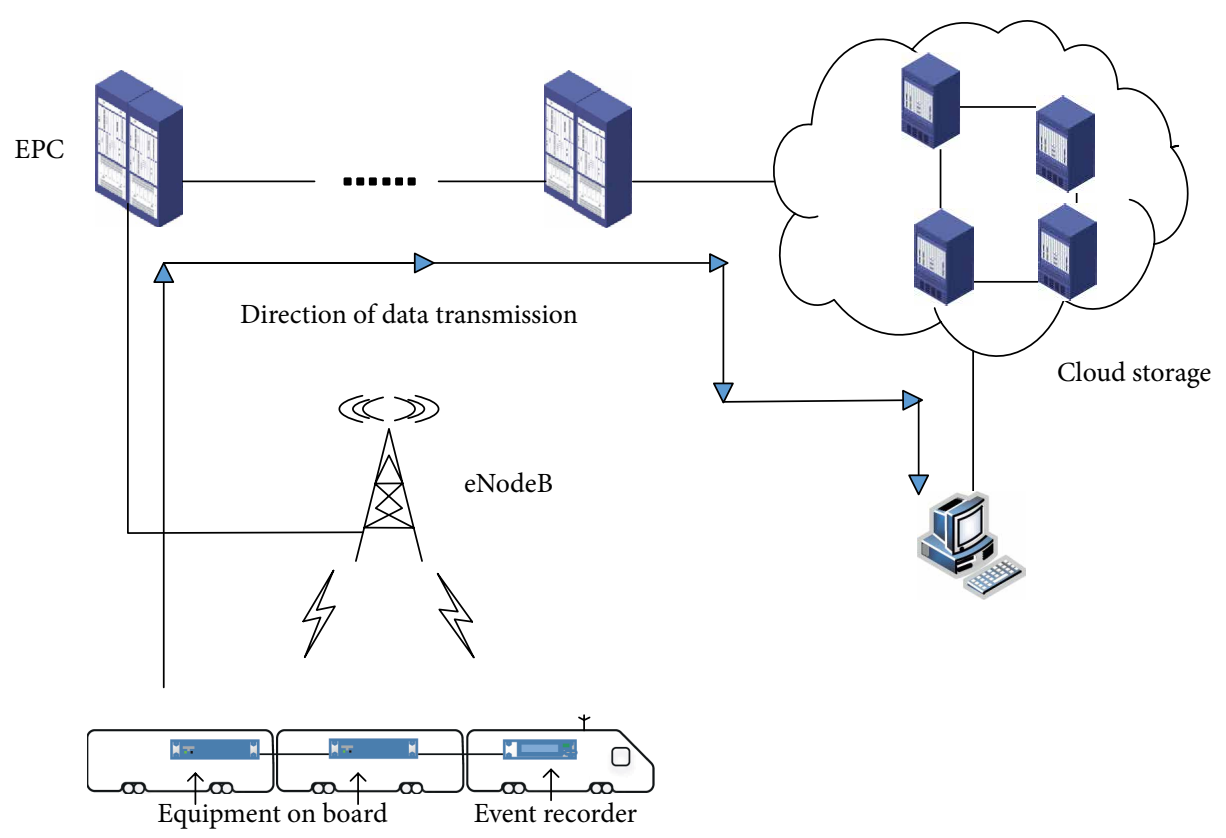

FIGURE 13: Data transmission process from the train to the cloud.

can check the train running condition and equipment status in real time, and analyze whether the train is in a normal state according to the existing experience and existing analysis methods to ensure the safe operation of the train.

Cloud storage is a model of online storage. Information is stored on the Internet, and capacity is provided on demand, enabling fast, persistent, and anytime, anywhere access to data. From the perspective of information storage, cloud storage has substantial advantages, including unlimited storage space, so the information of various states during train operation can be saved, providing researchers with more comprehensive and detailed data parameters, which is conducive to the simulation of train operation process and analysis of train operation fault by employees. On the other hand, in terms of the reliability of information storage, cloud storage adopts a third-party platform, and the stored data will not be lost or damaged due to the failure of the railway system.

LTE communication technology can guarantee a high data transmission rate. Therefore, it is incredibly reliable to use LTE-based communication technology to transmit the information from the event recorder to the cloud in real time. By using LTE wireless communication technology, the data is uploaded to the cloud in real time, and the staff can access the corresponding modules according to their needs and get the latest data at any time.

As shown in Figure 13, the event recorder obtains the information of equipment in each carriage and relevant data of running state, which is stored in the event recording memory. In the process of train operation, the train is always under the coverage of the LTE communication network. The train transfers the in-memory information to the cloud at any time and anywhere through one or more core networks, to realize high-capacity and highly reliable data storage.

\section{Conclusions}

In this paper, we have introduced the urban rail transit CBTC systems and train ground communication systems. According to communication performance parameters, the reliability of the urban rail transit CBTC train communication system based on LTE was defined. Then, the reliability of the train ground communication system was calculated using delay and packet loss. Besides, we also proposed a new storage method of information of train using communication network. The results show that the train ground communication system using LTE technology can complete the information transmission of urban rail transit systems and it has high reliability.

\section{Data Availability}

The data used to support the findings of this study are owned by a company.

\section{Conflicts of Interest}

The authors declare that they have no conflicts of interest.

\section{Acknowledgments}

This study was supported by Beijing Jiaotong University (No. 2018JBZ006).

\section{References}

[1] IEEE, "1474.1-2004 - IEEE standard for communicationsbased train control (CBTC) performance and functional requirements," IEEE, pp. 1-45, 2005. 
[2] R. D. Pascoe and T. N. Eichorn, "What is communication-based train control?," Vehicular Technology Magazine IEEE, vol. 4, no. 4, pp. 16-21, 2009.

[3] X. J. Liu, Research on the key technologies of communication based train control system in urban rail transit, Lanzhou Jiaotong University, Lanzhou, 2009.

[4] W. Sun, F. R. Yu, T. Tang, and B. Bing, "Energy-efficient communication-based train control (CBTC) systems with random delay and packet drop," in IEEE Global Communications Conference, IEEE, pp. 1353-1357, Austin, TX, USA, 2015.

[5] C. Teng, L. Wang, and C. Si, "Design and performance tests in different test scenarios based on the TD-LTE train-ground communication system," in IEEE International Conference on Computer and Communications, IEEE, Chengdu, China, 2017.

[6] B. Bu, F. R. Yu, and T. Tang, "Method to improve the performance of communication-based train control (CBTC) systems with transmission delays and packet drops," in IEEE International Conference on Intelligent Transportation Systems, IEEE, Qingdao, China, pp. 762-767, 2014.

[7] Y. G. Wang and L. Zhu, "Handover performance test and analysis in TD-LTE based CBTC train ground communication systems," in Chinese Automation Congress (CAC), IEEE, Jinan, China, 2017.

[8] S. Sesia, I. Toufik, and M. Baker, "LTE-The UMTS Long Term Evolution: From Theory to Practice," Wiley, pp. 437-440, 2009.

[9] C. Teng, L. Wang, and C. Si, "Design and performance tests in different test scenarios based on the TD-LTE train-ground communication system," in IEEE International Conference on Computer and Communications, IEEE, Chengdu, China, 2017.

[10] IEEE, "IEEE Standard for Information TechnologyTelecommunications and Information Exchange Between Systems-Local and Metropolitan Area Networks-Specific Requirements-Part 11: Wireless LAN Medium Access," Control (MAC) and Physical Layer (PHY) Specifications[M]. IEEE, 2012.

[11] H. Zhao, L. Zhu, H. Jiang, and T. Tang, "Design and performance tests in an integrated TD-LTE based train ground communication system," in IEEE International Conference on Intelligent Transportation Systems, IEEE, Qingdao, China, pp. 747-750, 2014.

[12] X. Wang, H. Jiang, T. Tang, and H. Zhao, "The QoS analysis of train-ground communication system based on TD-LTE in urban rail transit," in IEEE International Conference on Intelligent Rail Transportation, IEEE, Birmingham, UK, pp. 49-54, 2016.

[13] D. B. Green and A. S. Obaidat, "An accurate line of sight propagation performance model for ad-hoc 802.11 wireless LAN (WLAN) devices", in IEEE International Conference on Communications, IEEE, NY, USA, vol. 5, pp. 3424-3428, 2002.

[14] H. Wang, F. R. Yu, and H. Jiang, "Modeling of radio channels with leaky coaxial cable for LTE-M based CBTC systems," IEEE Communications Letters, vol. 20, no. 5, pp. 1038-1041, 2016.

[15] L. Zhu, Y. Zhang, B. Ning, and H. Jiang, "Train-ground communication in CBTC based on $802.11 \mathrm{~b}$ : design and performance research," in WRI International Conference on Communications and Mobile Computing, IEEE, Yunnan, China, pp. 368-372, 2009.

[16] B. Bu, F. R. Yu, and T. Tang, "performance improved methods for communication-based train control systems with random packet drops," IEEE Transactions on Intelligent Transportation Systems, vol. 15, no. 3, pp. 1179-1192, 2014.

[17] Y. Meng, “CBTC system analysis," Wireless Internet Technology, 2015.
[18] J. D. Osteen, C. Gonzalez, and K. J. Sampson, "Research on the Key Techniques of the Independent and Innovative CBTC[J]," Urban Rapid Rail Transit, vol. 107, no. 52, pp. 22710-22715, 2011.

[19] L. Zhu, F. R. Yu, B. Ning, and T. Tang, "Cross-layer handoff design in mimo-enabled wlans for communication-based train control (CBTC) Systems," IEEE Journal on Selected Areas in Communications, vol. 30, no. 4, pp. 719-728, 2012.

[20] F. Bai and H. Krishnan, "Reliability analysis of DSRC wireless communication for vehicle safety applications," in IEEE Intelligent Transportation Systems Conference, IEEE, Toronto, Ont., Canada, pp. 355-362, 2006.

[21] X. Wang, L. Liu, T. Tang, and W. Sun, "Enhancing communication-based train control systems through trainto-train communications," IEEE Transactions on Intelligent Transportation Systems, vol. 20, no. 4, pp. 1544-1561, 2019.

[22] X. Wang, L. Liu, T. Tang, and L. Zhu, "Next generation traincentric communication-based train control system with trainto-train (T2T) communications," in International Conference on Intelligent Rail Transportation (ICIRT), IEEE, Singapore, pp. 1-5, 2018. 


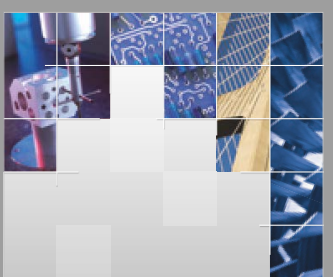

\section{Enfincering}
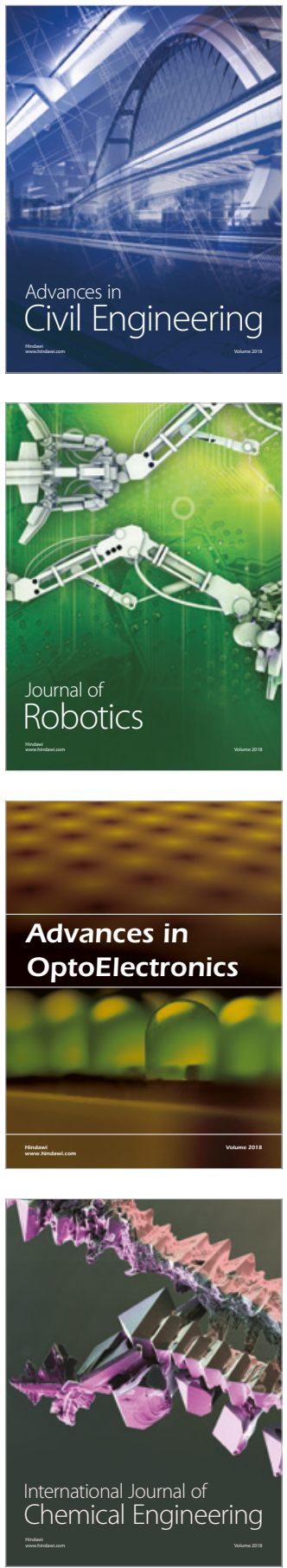

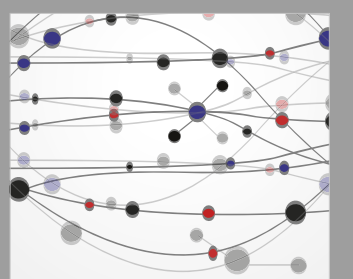

\section{Rotating \\ Machinery}

The Scientific World Journal

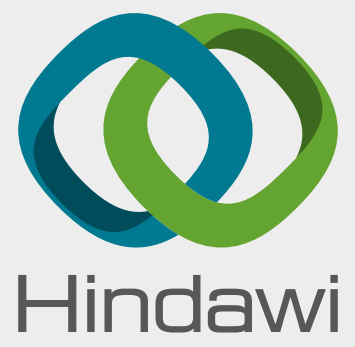

Submit your manuscripts at

www.hindawi.com
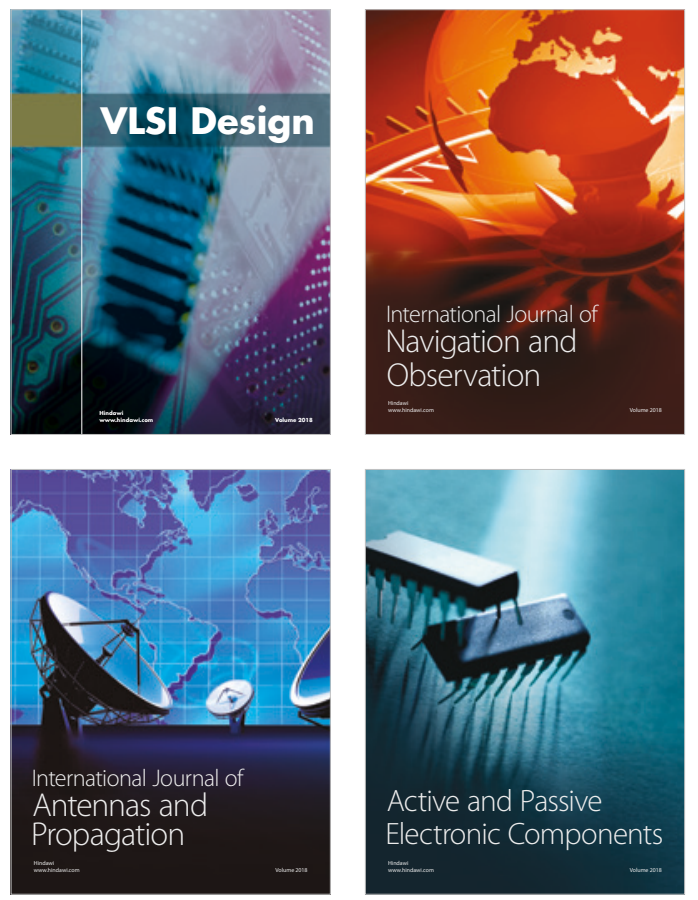
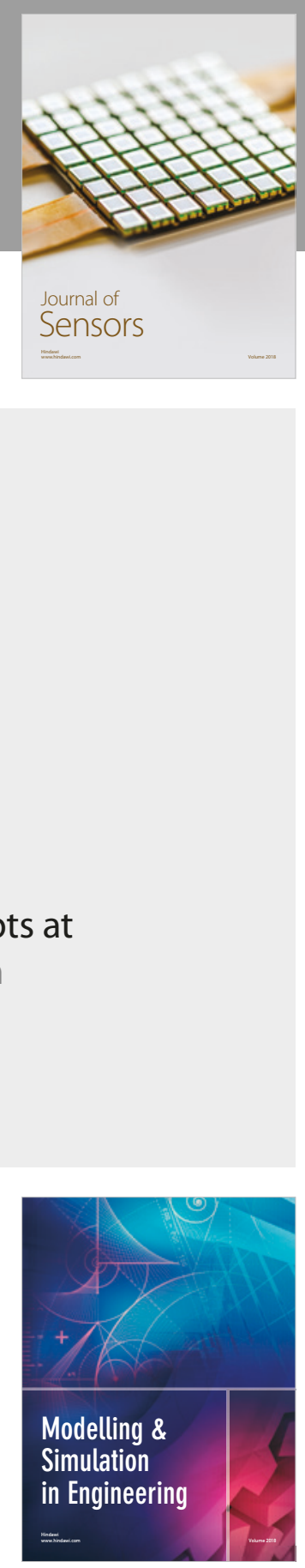

\section{Advances \\ Multimedia}
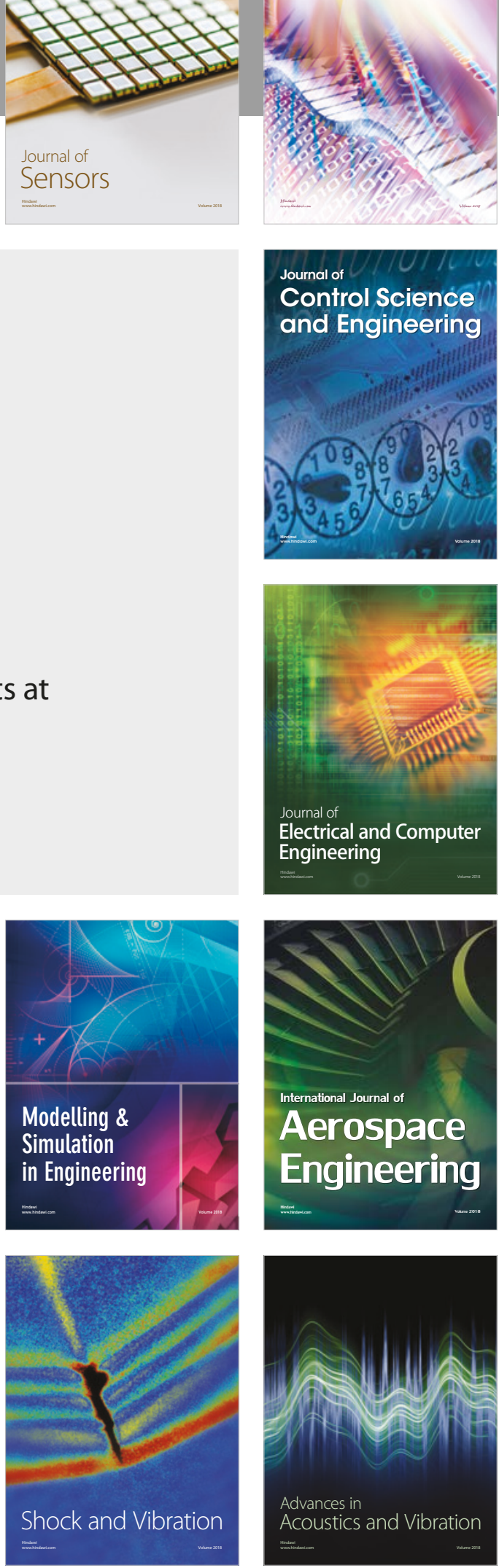